\title{
Combined intervention with pioglitazone and $n-3$ fatty acids in metformin-treated type 2 diabetic patients: improvement of lipid metabolism
}

Jiri Veleba', Jan Kopecky Jr. ', Petra Janovska², Ondrej Kuda², Olga Horakova², Hana Malinska', Ludmila Kazdova', Olena Oliyarnyk1, Vojtech Skop ${ }^{1}$, Jaroslava Trnovska', Milan Hajek', Antonin Skoch', Pavel Flachs², Kristina Bardova², Martin Rossmeisl ${ }^{2}$, Josune Olza ${ }^{3}$, Gabriela Salim de Castro ${ }^{3}$, Philip C. Calder ${ }^{3}$, Alzbeta Gardlo ${ }^{2,4}$, Eva Fiserova ${ }^{4}$, Jørgen Jensen ${ }^{5}$, Morten Bryhn $^{6}$, Jan Kopecky Sr. ${ }^{2^{*}}$ and Terezie Pelikanova ${ }^{1^{*}}$

\begin{abstract}
Background: The marine $n-3$ fatty acids, eicosapentaenoic acid (EPA) and docosahexaenoic acid (DHA) exert numerous beneficial effects on health, but their potency to improve treatment of type 2 diabetic (T2D) patients remains poorly characterized. We aimed to evaluate the effect of a combination intervention using EPA + DHA and the insulin-sensitizing drug pioglitazone in overweight/obese T2D patients already treated with metformin.
\end{abstract}

Methods: In a parallel-group, four-arm, randomized trial, 69 patients (66 \% men) were assigned to 24-week-intervention using: (i) corn oil (5 g/day; Placebo), (ii) pioglitazone (15 mg/day; Pio), (iii) EPA + DHA concentrate (5 g/day, containing $2.8 \mathrm{~g}$ EPA + DHA; Omega-3), or (iv) pioglitazone and EPA + DHA concentrate (Pio\& Omega-3). Data from 60 patients were used for the final evaluation. At baseline and after intervention, various metabolic markers, adiponectin and cytokines were evaluated in serum using standard procedures, EPA + DHA content in serum phospholipids was evaluated using shotgun lipidomics and mass spectrometry, and hyperinsulinemic-euglycemic clamp and meal test were also performed. Indirect calorimetry was conducted after the intervention. Primary endpoints were changes from baseline in insulin sensitivity evaluated using hyperinsulinemic-euglycemic clamp and in serum triacylglycerol concentrations in fasting state. Secondary endpoints included changes in fasting glycemia and glycated hemoglobin $\left(\mathrm{HbA}_{1 c}\right)$, changes in postprandial glucose, free fatty acid and triacylglycerol concentrations, metabolic flexibility assessed by indirect calorimetry, and inflammatory markers.

Results: Omega-3 and Pio\& Omega-3 increased EPA + DHA content in serum phospholipids. Pio and Pio\& Omega-3 increased body weight and adiponectin levels. Both fasting glycemia and $\mathrm{HbA}_{1 c}$ were increased by Omega-3, but were unchanged by Pio\& Omega-3. Insulin sensitivity was not affected by Omega-3, while it was improved by Pio\& Omega-3. Fasting triacylglycerol concentrations and inflammatory markers were not significantly affected by any of the interventions. Lipid metabolism in the meal test and metabolic flexibility were additively improved by Pio\& Omega-3.

(Continued on next page)

\footnotetext{
* Correspondence: kopecky@biomed.cas.cz; tepe@medicon.cz

${ }^{2}$ Department of Adipose Tissue Biology, Institute of Physiology of the Czech

Academy of Sciences, Prague, Czech Republic

${ }^{1}$ Institute for Clinical and Experimental Medicine, Prague, Czech Republic

Full list of author information is available at the end of the article
}

C Biomed Central

(c) 2015 Veleba et al. Open Access This article is distributed under the terms of the Creative Commons Attribution 4.0 International License (http://creativecommons.org/licenses/by/4.0/), which permits unrestricted use, distribution, and reproduction in any medium, provided you give appropriate credit to the original author(s) and the source, provide a link to the Creative Commons license, and indicate if changes were made. The Creative Commons Public Domain Dedication waiver (http://creativecommons.org/publicdomain/zero/1.0/) applies to the data made available in this article, unless otherwise stated. 
(Continued from previous page)

Conclusion: Besides preventing a modest negative effect of $n-3$ fatty acids on glycemic control, the combination of pioglitazone and EPA + DHA can be used to improve lipid metabolism in T2D patients on stable metformin therapy.

Trial registration: EudraCT number 2009-011106-42.

Keywords: Eicosapentaenoic acid, Docosahexaenoic acid, Indirect calorimetry, Meal test, Humans, Hyperinsulinemiceuglycemic clamp

\section{Background}

The complex etiology of type 2 diabetes (T2D) prompts for the use of a combination therapy to target multiple underlying mechanisms. Indeed, standards of medical care in diabetes recommend the combination therapy of metformin and other anti-diabetic drugs, next to metformin monotherapy [1]. Major effects of metformin include the lowering of hepatic glucose production and fasting glycemia and reduced risk of cardiovascular events [2], as well as an independent anti-inflammatory action [3] and amelioration of the oxidative stress [4]. The combination of metformin and the peroxisome proliferatoractivated receptor (PPAR) $\gamma$ agonist pioglitazone, a drug from the thiazolidinedione family, provided superior clinical outcomes to metformin alone [5]. In spite of the beneficial effects of thiazolidinediones on glycemic control, insulin sensitivity, inflammation and oxidative stress [2, 4, 5], as well as their triacylglycerol-lowering effect in both humans [5] and mice [6,7], clinical use of pioglitazone has declined recently due to the risk of its side-effects (reviewed in $[1,8]$ ). However, this risk could be outweighed by the benefits of pioglitazone in prevention of cardiovascular disease [9], the leading cause of death in patients with T2D [10]. Indeed, pioglitazone is associated with a relatively low risk of allcause mortality (reviewed in [8]).

Dietary interventions represent an important part of any management or treatment strategy for patients with T2D. Naturally occurring long-chain $n-3$ polyunsaturated fatty acids, namely eicosapentaenoic acid (EPA; 20:5 n-3) and docosahexaenoic acid (DHA; 22:6 $n-3)$, are considered to be healthy dietary constituents in diabetics [11]. These fatty acids exert anti-inflammatory and hypolipidemic effects, while increasing catabolism of lipids via a PPAR $\alpha$-mediated mechanism [11, 12]. At a daily dose of $4 \mathrm{~g}$, EPA + DHA are approved for the treatment of hypertriacylglycerolemia [13] and could ameliorate non-alcoholic fatty liver disease [14]. Even modest consumption of EPA + DHA (0.25-0.5 g/day) helps to prevent cardiovascular disease [15], reflecting probably the stabilization of atherosclerotic plaques [16]. The hypolipidemic effect of $n-3$ fatty acids could also be involved, since increased postprandial triacylglycerolemia represents an independent risk factor for cardiovascular disease in T2D patients $[10,17]$. In contrast with the earlier clinical trials, most of the recent studies did not show a benefit of EPA + DHA in the secondary prevention of cardiovascular disease (reviewed in [11]). Importantly, in patients with T2D within a large cohort, positive cardiovascular effects of $n-3$ fatty acids were observed [18]. Regarding the effects of EPA + DHA on glycemic control and insulin sensitivity, positive results have been obtained in animal models (reviewed in [11]), and in the prevention of T2D in obese children and young overweight/obese individuals [19]. Mixed results were obtained with respect to prevention of T2D by EPA + DHA in adult humans; in patients with T2D, either no or detrimental effects on glucose homeostasis were found in older studies, while more recent studies mostly showed neutral effects [11].

Our experiments in mice with diet-induced obesity $[6,7,20]$ demonstrated additive beneficial effects of a combined intervention using EPA + DHA and thiazolidinediones on insulin sensitivity, glucose tolerance, metabolic flexibility, lipid metabolism, hepatic steatosis, inflammation and obesity. These effects were observed in both the prevention $[6,7,20]$ and reversal [7] of obesity-associated phenotypes. Therefore, in this study, we sought to examine whether EPA + DHA, at a dose of $\sim 2.8$ g/day (i.e., 5 g EPA + DHA concentrate/day), could modulate the effects of pioglitazone in overweight and obese patients with T2D, specifically in well-controlled patients treated with metformin. In order to unmask potential additive effects, pioglitazone was used at a relatively low dose of $15 \mathrm{mg} /$ day, which is the initial recommended dose for treatment. The major goal of the study was to characterize the effect of the combined intervention on glucose homeostasis and lipid metabolism.

\section{Materials and methods Study design and patients}

A 24-week, parallel-group, four-arm, randomized trial (EudraCT number 2009-011106-42) was conducted in accordance with the principles of the Declaration of Helsinki (2008 revision) and with approval by the Institutional Ethical Committee. All patients provided written informed consent prior to their participation.

Inclusion criteria were 40-70 years of age, diagnosis of T2D as defined by the criteria of the American Diabetes 
Association and recognized by WHO, Expert Committee on the Diagnosis and Classification of Diabetes Mellitus (American Diabetes Association, 2004) diagnosed at least 3 months preceding screening visit, treatment by oral metformin as a monotherapy at a stable dose (0.5-3.0 g/day) for at least 1 month and no other antidiabetic agent, hemoglobin $\mathrm{A}_{1 \mathrm{c}}\left(\mathrm{HbA}_{1 \mathrm{c}}\right)<80 \mathrm{mmol} /$ mol, fasting plasma triacylglycerols $\leq 6 \mathrm{mmol} / \mathrm{l}$, BMI $25-45 \mathrm{~kg} / \mathrm{m}^{2}$, ability and willingness to adhere to the protocol and signed and dated written Informed consent obtained before any trial-related activities. Exclusion criteria were type 1 diabetes, uncorrected thyroid dysfunction, significant weight gain or loss $(>5 \%$ of total body weight within the past 3 months), therapy with insulin, or warfarin or fibrates within past 3 months (statins and salicylic acid were allowed; $51 \%$ of patients were treated with either simvastatin or atorvastatin), tachycardia ( $>100$ beats/min; or use of stable doses of antihypertensives shorter than 3 months prior the screening and during the trial), history of cardiovascular disease (myocardial infarction in the last year, coronary revascularization including percutaneous transluminal coronary angioplasty, coronary artery bypass graft surgery in the previous year and no subsequent angina, unstable angina, congestive heart failure), pregnancy or lactation, significant renal impairment (serum creatinine $>150 \mu \mathrm{mol} / \mathrm{l}$ ), chronic or advanced hepato-biliary diseases, history of alcohol or substance abuse within the past year, allergy to any of the capsule excipients, participation in any other clinical trial during the previous 3 months, and clinically significant anemia (hemoglobin $<120 \mathrm{~g} / \mathrm{l}$ for males and $<110 \mathrm{~g} / \mathrm{l}$ for females) or any other abnormal hemoglobin profile.

\section{Procedures}

Out of 294 patients subjected to an initial screening, 69 eligible patients (66 \% men) were enrolled (Fig. 1) at the Diabetes Centre, Institute for Clinical and Experimental Medicine, Prague, Czech Republic. All measurements, procedures and sample collection were performed at week 0 and week 24 (2 visits during each week), on an outpatient basis, after overnight $(8-10 \mathrm{~h})$ fasting with water ad libitum. During week 0 (baseline; 3 weeks after the screening visit maximum), at the first visit, serum and muscle samples were collected (see below and Additional file 1), and a hyperinsulinemic-euglycemic clamp was performed (see below). At the second visit one week later, a standard meal test was performed (see below), followed by proton magnetic resonance spectroscopy (Magnetom Trio, Siemens, Erlangen, Germany) of liver and skeletal muscle to measure lipid content as described in Additional file 1. At the second visit, patients were randomized to (i) $5 \mathrm{~g} /$ day corn oil (Placebo), (ii) $15 \mathrm{mg} /$ day pioglitazone (Pio; Actos, Takeda), (iii) 5 g/day EPA + DHA concentrate (Omega-3; EPAX 1050TG, EPAX AS, containing about $15 \%$ EPA, $40 \%$ DHA, wt/wt; i.e., $2.8 \mathrm{~g}$ EPA + DHA), and (iv) the combination of pioglitazone with EPAX 1050TG (Pio\& Omega-3). Randomization was performed using a computer-based algorithm arranging experimental units in blocks of four. The randomization code was kept secret and revealed after the clean-file procedure

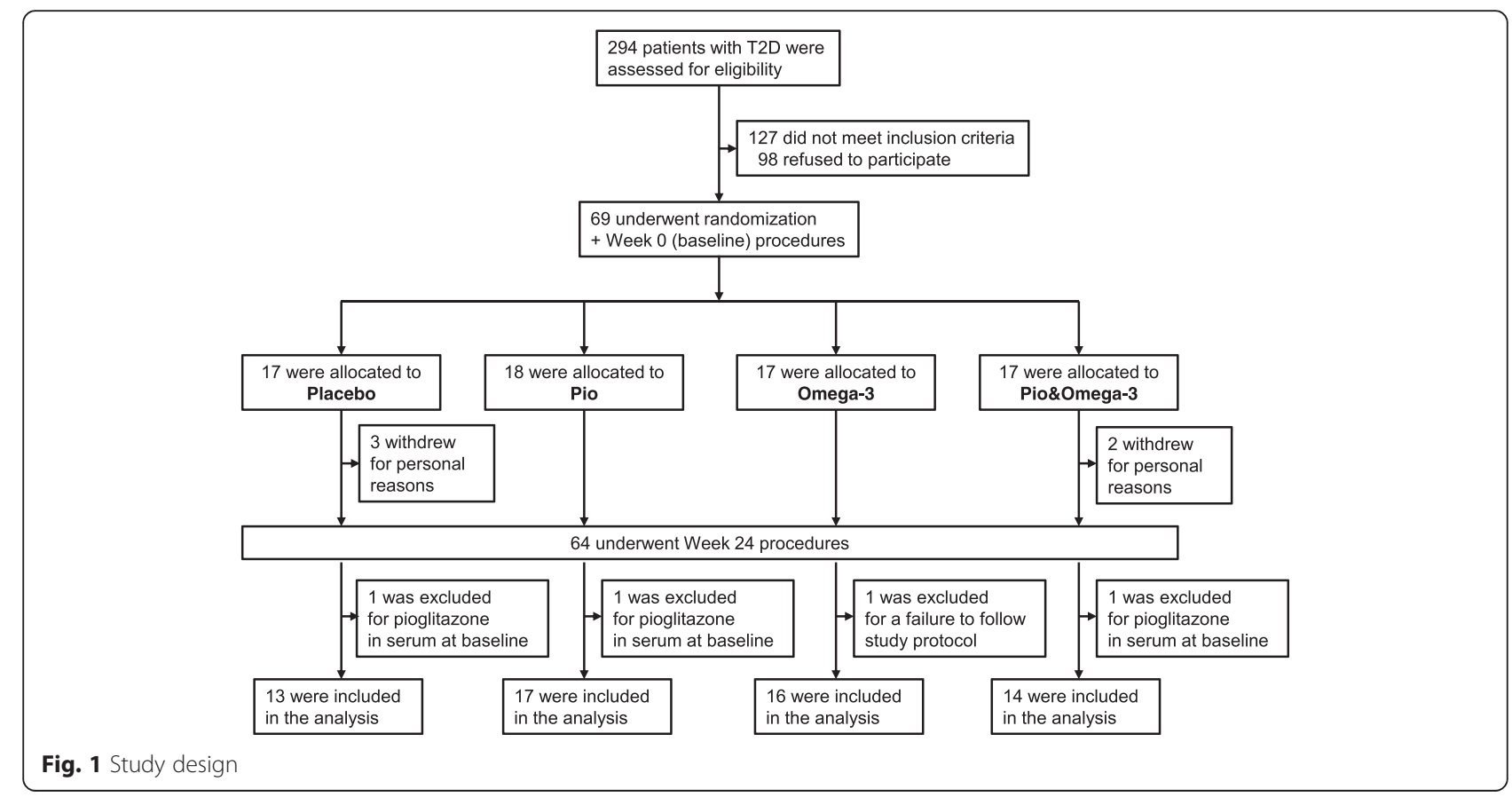


had been completed when all data had been filled in the case report forms. Placebo and Omega-3 were administered as gelatin-coated $1 \mathrm{~g}$ capsules. Thus, the study was double blind for EPA + DHA and open-label for pioglitazone. During week 24, patients were handled similarly as during week 0 , except for also performing indirect calorimetry in conjunction with the clamp (see below).

\section{Anthropometric measurements}

Body weight (and height, data not shown) was measured using periodically calibrated scales accurate to $0.1 \mathrm{~kg}$. Waist circumference was measured with a measuring tape placed at the midpoint between the lowest rib and the upper part of the iliac bone (results not shown). Body mass index (BMI) was calculated using the Quetelet formula (weight in kilograms divided by the square of the body height). Blood pressure was measured after $5 \mathrm{~min}$ in a seated position at rest, using a digital M6 Comfort monitor (Omron, Kyoto, Japan). Three measurements were taken 2 min apart. The first measurement was discarded, and the mean of the remaining two measurements was recorded.

\section{Hyperinsulinemic-euglycemic clamp}

A $3 \mathrm{~h}$ clamp $\left(1 \mathrm{mU} / \mathrm{kg} \cdot \mathrm{min}^{-1}\right)$, was conducted as described previously [21]. A teflon cannula (Venflon; Viggo, Helsingborg, Sweden) was inserted into an antecubital vein for the infusion of all test substances. A second cannula was inserted into a wrist vein for blood sampling and the hand was placed in a heated $\left(65{ }^{\circ} \mathrm{C}\right)$ box to achieve venous blood arterialization. A stepwise primed-continuous insulin infusion $(1 \mathrm{mU} / \mathrm{kg}$ body weight.min ${ }^{-1}$ of Actrapid HM; NovoNordisk, Copenhagen, Denmark) was administrated to acutely raise and maintain the plasma concentration of insulin at $\sim 75 \mu \mathrm{U} / \mathrm{ml}$. Glycemia during the clamps was maintained at approx. $5.5 \mathrm{mmol} / \mathrm{l}$ by continuous infusion of $15 \%$ glucose. Arterialized blood glucose concentration was determined every 5-10 min as described in Other analytical methods (see below) and the infusion rate was adjusted accordingly. Mean plasma glucose concentrations were comparable within the groups during clamps before and after 24 weeks. The coefficients of variation of glycemia during the studies were less than $5 \%$. Insulin sensitivity was estimated as the glucose disposal rate (M), i.e. the amount of glucose ( $\mathrm{mg} / \mathrm{kg}$ body weight. $\left.\mathrm{min}^{-1}\right)$ needed to maintain the concentration of glucose during the last $20 \mathrm{~min}$ of the clamp.

\section{Indirect calorimetry}

At week 24, indirect calorimetry was conducted for $30 \mathrm{~min}$ (basal values in fasting state) just before and during the last $30 \mathrm{~min}$ of the clamp, using an open-circuit system (VMAX; SensorMedics, Anaheim, CA, USA).
Oxygen consumption $\left(\mathrm{VO}_{2} ; \mathrm{ml} \mathrm{O}_{2} / \mathrm{min}\right)$ and carbon dioxide production $\left(V \mathrm{CO}_{2} ; \mathrm{ml} \mathrm{CO} \mathrm{CO}_{2} / \mathrm{min}\right)$ were recorded every $1 \mathrm{~min}$. To assess fuel partitioning, respiratory quotient (RQ; $\mathrm{RQ}=V \mathrm{CO}_{2} / V \mathrm{O}_{2}$ ) was estimated and substrate utilization and resting energy expenditure (REE) were calculated. Non-oxidative glucose disposal rate (GDR) was calculated by subtracting the rate of glucose oxidation from the total rate of glucose uptake during the last 20 min of the clamp [22].

\section{Meal test}

A meal test was performed as before [21]. After an overnight fast, subjects received a standard breakfast (baguette Crocodille Cheese Gourmet: 180 g, energy $452.8 \mathrm{kcal}(1895.7 \mathrm{~kJ}))$ of the following composition: carbohydrates $49 \mathrm{~g}$ (45 \% energy), proteins $18.5 \mathrm{~g}$ (17 \% energy), lipids 18.8 g (38 \% energy), of which saturated fatty acids $6.8 \mathrm{~g}$, monounsaturated fatty acids $6.0 \mathrm{~g}$, and polyunsaturated fatty acids $5.0 \mathrm{~g}$. Serum concentrations of glucose, immunoreactive insulin, C-peptide, non-esterified fatty acids (NEFA), and triacylglycerol were measured at 0, 30, 60 and 120 min (see below in Other analytical methods). Data were expressed as area under the curve (AUC).

\section{Content of selected fatty acids in serum phospholipids}

Serum samples from fasted patients were analyzed using shotgun lipidomics and mass spectrometry, and a sum of concentrations of phospholipids containing EPA and/or DHA divided by total concentration of all phospholipids (Omega-3 PhL Index) was used as a biomarker of EPA and DHA status. Briefly, $50 \mu \mathrm{l}$-aliquots of serum were transferred into disposable borosilicate glass tubes with $100 \mu \mathrm{l}$ of methanol/butylated hydroxytoluene $(1,000: 1 ; \mathrm{v} / \mathrm{v})$ containing internal standards for quantification of lipid species: 17:0-17:0 phosphatidic acid, phosphatidylethanolamine, phosphatidylcholine, phosphatidylglycerol, phosphatidylserine, and phosphatidylinositol, respectively (75 $\mathrm{nM}$ final concentration). Lipid extracts were prepared using a modified procedure of Bligh and Dyer as previously described [23]. Each lipid extract was diluted with dichloromethane/metha$\mathrm{nol} /$ isopropanol (1:2:4, $\mathrm{v} / \mathrm{v} / \mathrm{v}$ and $5 \mathrm{mM}$ ammonium acetate) prior to infusion into a mass spectrometer (MS; QTRAP 5500, Sciex, USA; equipped with Turbo V ESI) for the analysis of phospholipids. All the mass spectra and tandem mass spectra were automatically acquired using multiplexed precursor ion (PIS) and neutral loss (NL) scans in positive and negative mode [23]. Analyst 1.6.1/Lipidview 1.3 software was used to identify molecular species and to determine amounts of individual lipids based on internal standard concentrations assuming comparable ionization of standards and phospholipids. Sum formula annotation (e.g. PE 34:2) 
and acyl chain information coming from negative PIS (e.g. PE 34:2 - PIS 18:2) was used to calculate the additional acyl chain (e.g. PE 34:2=18:2+16:0). Only combinations of the major fatty acids (carbon:double bonds $-0: 0,12: 0,14: 0,14: 1,16: 0,16: 1,18: 0,18: 1$, $18: 2,18: 3,20: 0,20: 1,20: 2,20: 3,20: 4,20: 5,22: 4,22: 5$, 22:6) were used for further data processing. The content of linoleic acid (LA; 18:2 n-6) in serum phospholipids was also determined.

\section{Other analytical methods}

Serum glucose levels were analyzed using the Beckman Analyser glucose-oxidase method (Beckman Instruments, Fullerton, CA, USA), plasma immunoreactive insulin and $\mathrm{C}$-peptide concentrations were determined using insulin and C-peptide IRMA kits (Immunotech, Prague, Czech Republic), $\mathrm{HbA}_{1 \mathrm{c}}$, was measured by HPLC (Tosoh, Tokyo, Japan), lipid concentrations were assessed by enzymatic methods (Roche, Basel, Switzerland) and HDL-cholesterol was measured after double precipitation with dextran and $\mathrm{MgCl}_{2}$ as described previously [24]. To assess oxidative stress, the amount of lipid peroxidation was determined as thiobarbituric acid reactive substances (TBARS) by the reaction with thiobarbituric acid, the whole blood level of reduced (GSH) and oxidized (GSSG) glutathione was determined with glutathione HPLC diagnostic kit (Chromsystems, Munich, Germany), and the activity of superoxide dismutase (SOD) was analyzed using a superoxide dismutase assay kit (Cayman Chemical, MI, USA). In fasting patients (Table 1), concentrations of serum cytokines were measured using ELISA kits from Biovendor (Czech Republic; total adiponectin, leptin), in the postabsortive state, various cytokines were analyzed by microbead Luminex ${ }^{\circ}$ assay (Luminex Corporation, Texas, United States; see Additional file 3). Serum pioglitazone levels were estimated using mass spectrometry (see above) [25].

\section{Study endpoints}

The primary endpoints were changes from baseline in (i) insulin sensitivity ( $M$; hyperinsulinemic-euglycemic clamp) and (ii) fasting triacylglycerol levels at week 24. Secondary endpoints included the changes in fasting glycemia and $\mathrm{HbA}_{1 \mathrm{c}}$, and postprandial change in glucose, NEFA and triacylglycerol levels (meal test), metabolic flexibility assessed by indirect calorimetry, and inflammatory markers.

\section{Statistical analysis}

A power calculation indicated that 16 patients were needed to detect a $7 \%$ difference in $\mathrm{M}$ due to the intervention with the probability 1 at the 0.05 level of significance (assuming accuracy of measurement $5 \%$ ). All values are presented as median and interquartile range
(IQR). Data from the baseline and the end of the study, and the changes $(\Delta)$ between the baseline and the end at week 24, were analyzed by the Kruskal-Wallis one-way analysis of variance (ANOVA) using SigmaStat 3.5 (SSI, San Jose, CA, USA) and the statistical software R version 3.1.0 (http://www.r-project.org). The HolmBonferroni corrections for multiple comparisons were used. Wilcoxon signed-rank test was used to analyze the effect of intervention within each subgroup. Threshold of significance was defined at a $p$ value of $\leq 0.05$. For the analysis of the dependence of the response ( $\Delta$ value) of selected variables on the corresponding change in Omega-3 $\mathrm{PhL}$ Index ( $\Delta$ Omega-3 PhL Index), a linear regression model with dummy variables that indicate a subgroup Placebo, Pio, Omega-3, and Pio\& Omega-3, respectively, was used. Models were considered with interactions since the effect of $\Delta$ Omega-3 PhL Index varies by subgroups, so $\Delta$ Omega-3 $\mathrm{PhL}$ Index and subgroups interact in affecting $\Delta$ value of selected variables.

\section{Results}

\section{Basal characteristics}

Of the 69 patients enrolled, data from 60 patients could be used for the final evaluation (Fig. 1). Thus, 5 patients withdrew owing to personal reasons; and after the intervention started, 1 patient was excluded due to failure to adhere to the study protocol. Based on serum pioglitazone measurements (not shown), 3 patients were excluded due to detection of pioglitazone already at the baseline, while the pioglitazone levels assessed at week 24 confirmed adherence to the study protocol in the Pio and Pio\& Omega-3 subgroups.

No significant differences were observed between the subgroups in basic anthropometric and biochemical characteristics measured in the fasting state during the study (Table 1). In the subgroup analysis by the effect of the 24-week-intervention (see $\Delta$-values in Table 1), both Pio and Pio\& Omega-3 increased body weight and BMI compared with Placebo or Omega-3 (except for Placebo vs. Pio in the case of body weight; $p=0.10$ ). Triacylglycerol, NEFA and total cholesterol in serum, blood lipoproteins including HDL cholesterol and LDL cholesterol, and markers of oxidative stress in serum including SOD activity, TBARS and GSSG/GSH ratio were not significantly affected by any of the interventions (Table 1 ). Magnetic resonance spectroscopy did not show any significant effect of the interventions on the ectopic lipid content (see Additional file 1). Leptin levels were increased in Pio compared with the other subgroups $(p=0.02)$. At week 24 , adiponectin levels were higher in Pio and Pio\& Omega-3 than in Placebo subgroup, reflecting $~ 1.6$-fold fold stimulatory effect in the median concentration in both Pio and Pio\& Omega-3 $(p<0.0001)$. Adiponectin levels in the Omega-3 subgroup were higher, 
Table 1 Basic anthropometric and biochemical characteristics of patients at baseline and after the interventions. The significance threshold for adjusted $p$-values using the Holm-Bonferroni correction is 0.05

\begin{tabular}{|c|c|c|c|c|}
\hline & Placebo & Pio & Omega-3 & Pio\& Omega-3 \\
\hline Age (y) & $62.0(58.0,65.0)$ & $62.0(60.0,65.0)$ & $59.5(55.8,63.8)$ & $60.5(55.3,65.5)$ \\
\hline \multicolumn{5}{|c|}{ Systolic blood pressure $(\mathrm{mmHg})$} \\
\hline Baseline & $138(129,149)$ & $142(136,151)$ & $146(135,153)$ & $139(131,145)$ \\
\hline Week 24 & $140(131,146)$ & $142(134,148)$ & $137(131,150)$ & $136(127,148)$ \\
\hline$\Delta$ & $2(-3,7)$ & $1(-5,3)$ & $-5(-8,2)$ & $-1(-8,1)$ \\
\hline$\Delta(\%)$ & $1(-2,5)$ & $1(-3,2)$ & $-3(-5,1)$ & $0(-6,1)$ \\
\hline \multicolumn{5}{|c|}{ Diastolic blood pressure (mmHg) } \\
\hline Baseline & $82(77,93)$ & $85(79,91)$ & $90(83,92)$ & $81(69,94)$ \\
\hline Week 24 & $85(70,90)$ & $82(73,89)$ & $86(75,90)$ & $82(76,92)$ \\
\hline$\Delta$ & $1(-6,6)$ & $-1(-9,2)$ & $0(-5,2)$ & $1(-7,5)$ \\
\hline$\Delta(\%)$ & $1(-8,7)$ & $-1(-8,2)$ & $0(-5,3)$ & $1(-8,7)$ \\
\hline \multicolumn{5}{|c|}{ Body weight (kg) } \\
\hline Baseline & $87.0(81.2,103.0)$ & $94.0(85.0,102.0)$ & $98.8(95.2,110.5)$ & $94.0(79.5,107.0)$ \\
\hline Week 24 & $84.0(79.0,103.0)$ & $95.0(88.0,103.0)$ & $97.5(91.5,108.3)$ & $95.5(83.0,111.5)$ \\
\hline$\Delta$ & $-1.0(-2.0,0.0)$ & $1.0(0.0,2.8)^{c}$ & $-1.5(-2.6,1.0)$ & $2.0(1.0,3.0)^{a, c}$ \\
\hline$\Delta(\%)$ & $-1.2(-2.4,0.0)$ & $1.3(0.0,2.3)^{c}$ & $-1.3(-2.7,0.8)$ & $1.9(1.0,4.2)^{a, c}$ \\
\hline \multicolumn{5}{|l|}{$\mathrm{BMI}\left(\mathrm{kg} / \mathrm{m}^{2}\right)$} \\
\hline Baseline & $30.9(27.7,33.5)$ & $32.0(29.6,35.4)$ & $34.0(29.2,37.5)$ & $31.4(27.9,39.8)$ \\
\hline Week 24 & $30.5(27.4,32.6)$ & $32.4(30.2,36.6)$ & $33.1(28.7,37.9)$ & $32.0(28.7,40.6)$ \\
\hline$\Delta$ & $-0.4(-0.8,0.0)$ & $0.4(0.0,0.9)^{a, c}$ & $-0.5(-1.0,0.3)$ & $0.7(0.4,1.2)^{a, c}$ \\
\hline$\Delta(\%)$ & $-1.4(-2.5,0.0)$ & $1.3(0.0,2.3)^{a, c}$ & $-1.3(-2.7,0.8)$ & $2.0(1.1,4.2)^{a, c}$ \\
\hline \multicolumn{5}{|c|}{ Triacylglycerol (mmol/l) } \\
\hline Baseline & $1.45(1.23,1.76)$ & $1.70(1.17,2.41)$ & $2.17(1.50,2.76)$ & $1.71(1.32,2.21)$ \\
\hline Week 24 & $1.41(1.10,2.28)$ & $1.89(1.24,2.36)$ & $1.67(1.53,2.31)$ & $1.52(1.06,2.05)$ \\
\hline$\Delta$ & $0.21(-0.13,0.48)$ & $0.13(-0.11,0.66)$ & $-0.48(-0.73,0.36)$ & $-0.14(-0.58,-0.02)$ \\
\hline$\Delta(\%)$ & $10.1(-10.6,31.3)$ & $4.9(-13.0,52.4)$ & $-18.2(-23.8,20.2)$ & $-12.2(-24.0,-1.1)$ \\
\hline \multicolumn{5}{|c|}{$\operatorname{NEFA}(\mathrm{mmol} / \mathrm{l})$} \\
\hline Baseline & $0.73(0.64,0.79)$ & $0.82(0.64,1.04)$ & $0.65(0.54,0.91)$ & $0.69(0.53,0.74)$ \\
\hline Week 24 & $0.65(0.51,0.87)$ & $0.51(0.37,0.79)$ & $0.60(0.47,0.85)$ & $0.53(0.31,0.70)$ \\
\hline$\Delta$ & $0.21(-0.13,0.48)$ & $0.13(-0.11,0.66)$ & $-0.48(-0.73,0.36)$ & $-0.14(-0.58,-0.02)$ \\
\hline$\Delta(\%)$ & $-11.0(-40.7,13.0)$ & $-43.2(-50.0,-15.4)$ & $-16.0(-47.6,25.7)$ & $-9.4(-43.0,18.7)$ \\
\hline \multicolumn{5}{|c|}{ Cholesterol total $(\mathrm{mmol} / \mathrm{l})$} \\
\hline Baseline & $4.60(4.00,5.60)$ & $4.54(3.70,5.50)$ & $4.70(4.49,5.37)$ & $5.25(4.78,5.95)$ \\
\hline Week 24 & $4.40(3.53,5.32)$ & $4.47(3.61,5.06)$ & $4.62(4.40,5.12)$ & $4.56(4.32,5.45)$ \\
\hline$\Delta$ & $-0.47(-0.62,-0.20)$ & $-0.07(-0.40,0.74)$ & $-0.13(-0.62,0.21)$ & $-0.23(-0.87,-0.04)$ \\
\hline$\Delta(\%)$ & $-9.8(-15.9,-4.3)$ & $-2.0(-7.8,13.5)$ & $-2.9(-12.9,4.5)$ & $-4.7(-16.4,-0.7)$ \\
\hline \multicolumn{5}{|c|}{ HDL cholesterol (mmol/l) } \\
\hline Baseline & $1.09(1.00,1.29)$ & $1.02(0.90,1.16)$ & $1.17(0.94,1.28)$ & $1.18(1.03,1.28)$ \\
\hline Week 24 & $1.04(0.79,1.17)$ & $1.07(0.96,1.18)$ & $1.04(0.91,1.25)$ & $1.24(1.10,1.58)$ \\
\hline$\Delta$ & $-0.05(-0.14,-0.04)$ & $0.07(-0.05,0.12)$ & $-0.04(-0.14,0.05)$ & $0.12(0.00,0.22)$ \\
\hline$\Delta(\%)$ & $-4.8(-10.9,-2.2)$ & $6.2(-6.5,8.8)$ & $-2.8(-14.4,4.4)$ & $10.5(0.9,19.2)$ \\
\hline \multicolumn{5}{|c|}{ LDL cholesterol $(\mathrm{mmol} / \mathrm{l})$} \\
\hline Baseline & $2.45(2.00,3.29)$ & $2.21(1.70,2.70)$ & $2.70(2.28,3.00)$ & $2.75(2.37,3.32)$ \\
\hline
\end{tabular}


Table 1 Basic anthropometric and biochemical characteristics of patients at baseline and after the interventions. The significance threshold for adjusted $p$-values using the Holm-Bonferroni correction is 0.05 (Continued)

\begin{tabular}{|c|c|c|c|c|}
\hline Week 24 & $2.60(1.90,3.02)$ & $2.60(1.86,2.94)$ & $2.81(2.56,3.24)$ & $3.03(2.33,3.53)$ \\
\hline$\Delta$ & $-0.09(-0.20,0.00)$ & $0.13(-0.03,0.41)$ & $0.16(-0.17,0.70)$ & $0.30(-0.17,0.65)$ \\
\hline$\Delta(\%)$ & $-4.4(-5.5,0.0)$ & $7.6(-1.2,16.6)$ & $6.8(-5.6,27.9)$ & $10.6(-7.7,20.2)$ \\
\hline \multicolumn{5}{|l|}{$\mathrm{SOD}(\mathrm{U} / \mathrm{ml})$} \\
\hline Baseline & $1.40(0.76,1.89)$ & $1.44(0.75,2.15)$ & $0.90(0.58,1.38)$ & $1.16(0.78,1.76)$ \\
\hline Week 24 & $1.22(0.99,1.76)$ & $1.11(0.86,1.99)$ & $0.90(0.70,1.35)$ & $0.88(0.48,1.25)$ \\
\hline$\Delta$ & $0.05(-0.67,0.29)$ & $0.21(-0.33,0.46)$ & $0.07(-0.25,0.46)$ & $-0.23(-0.68,0.23)$ \\
\hline$\Delta(\%)$ & $2.2(-33.4,38.5)$ & $24.3(-23.0,43.1)$ & $8.9(-22.0,46.1)$ & $-18.0(-49.5,34.8)$ \\
\hline \multicolumn{5}{|c|}{ TBARS (mmol/l) } \\
\hline Baseline & $1.71(1.33,2.04)$ & $1.40(1.16,2.14)$ & $1.46(1.35,2.29)$ & $1.64(1.26,2.10)$ \\
\hline Week 24 & $1.42(1.00,1.64)$ & $1.36(0.97,1.75)$ & $1.12(0.76,1.43)$ & $1.28(0.97,1.47)$ \\
\hline$\Delta$ & $-0.23(-0.76,0.28)$ & $-0.10(-0.42,0.05)$ & $-0.59(-0.93,0.12)$ & $-0.20(-0.78,0.09)$ \\
\hline$\Delta(\%)$ & $-17.5(-34.5,25.8)$ & $-6.7(-22.6,3.2)$ & $-43.0(-56.4,11.9)$ & $-10.8(-43.9,11.2)$ \\
\hline \multicolumn{5}{|c|}{ Ratio GSSG/GSH } \\
\hline Baseline & $0.17(0.12,0.20)$ & $0.14(0.11,0.17)$ & $0.11(0.09,0.15)$ & $0.11(0.08,0.16)$ \\
\hline Week 24 & $0.11(0.11,0.15)$ & $0.13(0.10,0.14)$ & $0.12(0.08,0.18)$ & $0.14(0.11,0.20)$ \\
\hline$\Delta$ & $-0.04(-0.09,0.02)$ & $0.00(-0.08,0.03)$ & $0.00(-0.02,0.03)$ & $0.01(-0.02,0.06)$ \\
\hline$\Delta(\%)$ & $-20.9(-47.5,19.9)$ & $-3.4(-48.1,67.3)$ & $2.1(-19.2,38.7)$ & $9.0(-12.7,53.2)$ \\
\hline \multicolumn{5}{|c|}{ Leptin (ng/ml) } \\
\hline Baseline & $13.2(10.6,17.2)$ & $12.6(10.0,26.1)$ & $12.7(7.0,23.9)$ & $21.7(9.7,30.4)$ \\
\hline Week 24 & $14.4(7.8,15.8)$ & $13.0(9.2,33.3)$ & $11.3(7.9,21.7)$ & $21.0(8.5,41.3)$ \\
\hline$\Delta$ & $-0.8(-1.8,0.6)$ & $2.4(0.5,3.0)$ & $-1.1(-2.9,1.0)$ & $-0.3(-1.1,0.5)$ \\
\hline$\Delta(\%)$ & $-3.2(-14.5,6.8)$ & $10.3(1.4,29.4)$ & $-9.9(-20.9,12.2)$ & $-1.9(-3.8,3.5)$ \\
\hline \multicolumn{5}{|c|}{ Adiponectin $(\mu \mathrm{g} / \mathrm{ml})$} \\
\hline Baseline & $6.3(5.6,7.3)$ & $5.7(4.6,6.9)$ & $5.3(4.7,5.9)$ & $5.6(4.8,6.4)$ \\
\hline Week 24 & $6.3(4.9,9.0)$ & $9.1(7.4,12.8)^{a, c}$ & $6.4(5.7,7.2)$ & $8.9(7.5,9.9)^{a, c}$ \\
\hline$\Delta$ & $0.4(0.0,1.0)$ & $3.5(2.6,5.0)^{a_{1} c}$ & $0.9(0.6,1.2)$ & $3.7(2.6,4.8)^{a, c}$ \\
\hline$\Delta(\%)$ & $9.4(-0.2,27.4)$ & $60.0(45.4,73.4)^{a, c}$ & $15.8(9.0,26.9)$ & $64.7(44.6,91.8)^{a, c}$ \\
\hline \multicolumn{5}{|c|}{ Omega-3 PhL Index } \\
\hline Baseline & $4.9(4.3,5.8)$ & $5.2(4.4,5.8)$ & $4.9(4.6,5.5)$ & $5.3(4.1,5.7)$ \\
\hline Week 24 & $4.4(4.1,4.8)$ & $4.5(4.2,5.2)$ & $8.2(7.1,8.9)^{a, b}$ & $8.3(7.4,9.2)^{a, b}$ \\
\hline$\Delta$ & $-0.1(-0.5,0.2)$ & $-0.3(-0.7,0.2)$ & $2.8(2.1,4.4)^{a, b}$ & $3.4(2.5,3.6)^{a, b}$ \\
\hline$\Delta(\%)$ & $-2.6(-10.5,4.6)$ & $-5.2(-13.9,2.7)$ & $60.8(43.9,88.5)^{\mathrm{a}, \mathrm{b}}$ & $62.8(41.4,99.9)^{a, b}$ \\
\hline
\end{tabular}

Data represent a median and interquartile range (Q1, Q3). Various parameters were analyzed in overnight (8-10-h) fasting patients at baseline and at week 24 of the respective interventions. $\Delta$, a difference between week 24 and baseline values; $\Delta$ (\%), a difference between week 24 and baseline values in \% of the baseline value; BW, body weight; BMI, body mass index, NEFA, non-esterified fatty acids; SOD, superoxide dismutase; TBARS, thiobarbituric acid reactive substances; GSSH, oxidized glutathione; GSH, reduced glutathione. ${ }^{a, b}$, cSignificant differences compared with Placebo, Pio, and Omega-3, respectively

reflecting 1.2-fold increase in the median in this subgroup $(p<0.001)$ but were unchanged in the Placebo subgroup (Table 1).

Omega-3 PhL Index was similar in all subgroups at the baseline, while at week 24 it was not affected by either Placebo or Pio, but increased to a similar extent (1.6-1.7-fold; $p<0.0001$ and $p=0.0001$, respectively) in response to both Omega-3 and Pio\& Omega-3 (Table 1). As observed before [26], Omega-3 PhL Index differed between individuals, showing up to $\sim 3$-fold differences when all 60 patients were compared at the baseline (Fig. 2 a-d). In the EPA + DHA supplemented subgroups (Omega-3 or Pio\& Omega-3) a maximum $\sim 2.2$-fold difference in the median Omega-3 PhL Index was observed between individuals at the end of the intervention (Table 1). The variable increase in the Omega-3 PhL Index in response to EPA + DHA supplementation was independent of the pre-intervention value (Fig. 2 c, d). 


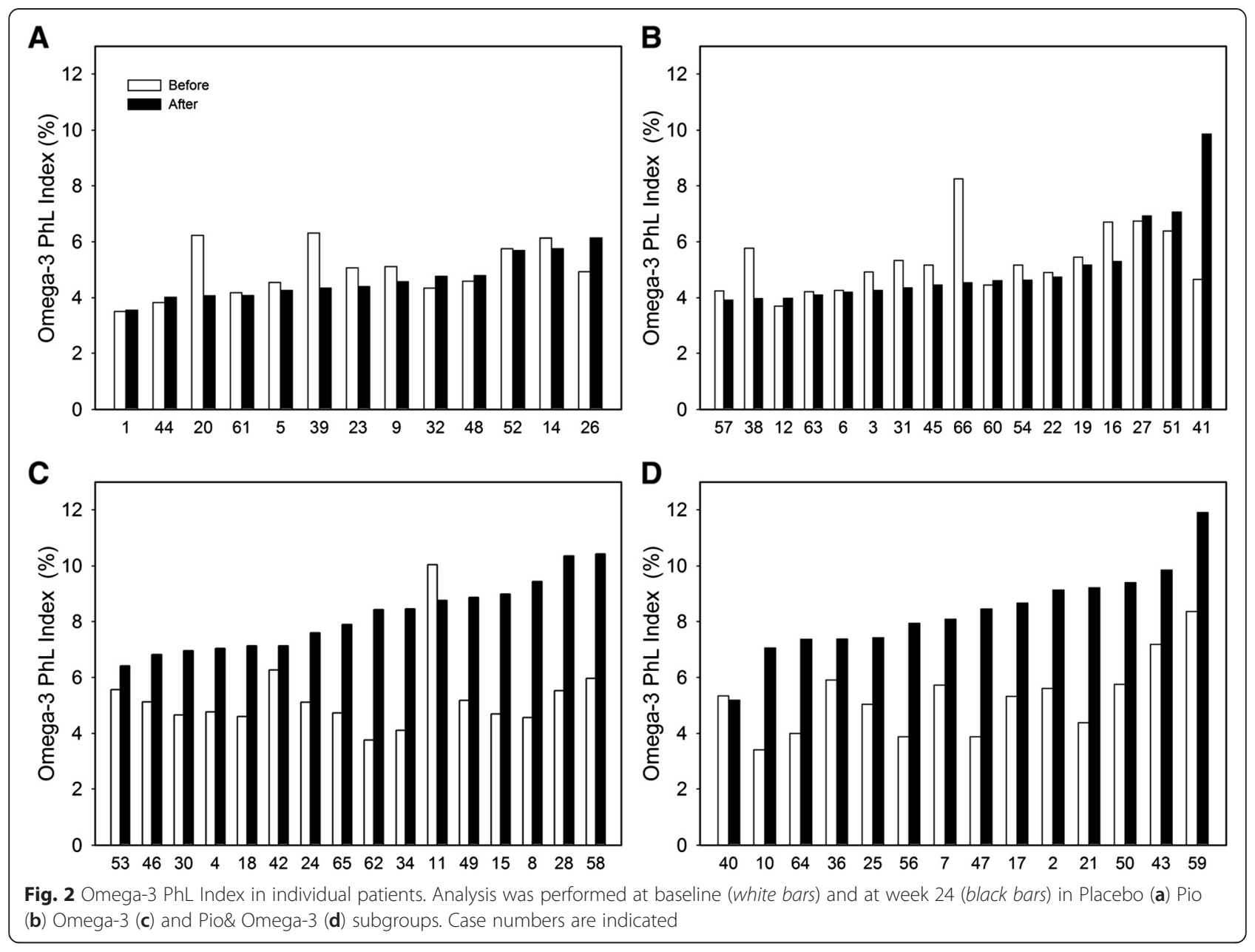

In contrast with the Omega-3 PhL Index (i.e., the EPA and DHA in serum phospolipids), LA content in phospholipids was not affected by any intervention and no differences in LA content between the subgroups of patients were found, either before or after the intervention (see Additional file 2).

\section{Glucose metabolism}

No differences between Placebo, Pio and Pio\& Omega-3 subgroups were observed in the markers of acute and long-term glycemic control, i.e., fasting blood glucose and serum $\mathrm{HbA}_{1 \mathrm{c}}$ level, respectively, either at baseline, or at week 24. (Table 2). In response to Omega-3, both parameters increased by $\sim 1$.2-fold (fasting blood glucose, $\left.p=0.02 ; \mathrm{HbA}_{1 \mathrm{c}}, p=0.01\right)$ resulting in a significant effect of Omega-3 (see $\Delta$-values in Table 2) and indicating a marginal deterioration of glycemic control. Glucose disposal rate, assessed using hyperinsulinemic-euglycemic clamp ( $M$ value), is a measure of insulin sensitivity. While it was similar in all the subgroups at baseline, $M$ increased $(p=0.04)$ in Pio\& Omega-3 subgroup during the intervention and at week 24 , it was higher in the
Pio\& Omega-3 compared with the Omega-3 subgroup. In the subgroup analysis by the effect of the intervention (see $\Delta$-values in Table 2), the effect of Pio $(p=0.12)$ and Pio\& Omega-3 $(p=0.12)$ tended to be different from that of Omega-3. Thus, regarding the effects on insulin sensitivity, Omega-3 exerted a neutral effect compared with Placebo, while the results collectively document a marginal improvement by the Pio\& Omega-3 intervention.

\section{Energy metabolism and metabolic flexibility}

At week 24, indirect calorimetry was performed in conjunction with the hyperinsulinemic-euglycemic clamp. None of the measured parameters, namely REE, RQ, carbohydrate oxidation and fat oxidation were significantly different between the subgroups before or during the clamp. Non-oxidative GDR, assessed during the clamp, was also similar in all subgroups (Table 3 ).

Next, we attempted to detect possible differences in metabolic flexibility between the subgroups at week 24 . We focused on the increase in RQ during the clamp, as a common way for assessment of metabolic flexibility to 
Table 2 Glucose homeostasis and insulin sensitivity at baseline and after the intervention. The significance threshold for adjusted p-values using the Holm-Bonferroni correction is 0.05

\begin{tabular}{|c|c|c|c|c|}
\hline & Placebo & Pio & Omega-3 & Pio\& Omega-3 \\
\hline \multicolumn{5}{|c|}{$\mathrm{HbA}_{1 \mathrm{c}}(\mathrm{IFCC}, \mathrm{mmol} / \mathrm{mol})$} \\
\hline Baseline & $52(48,56)$ & $52(47,54)$ & $50(47,55)$ & $49(44,53)$ \\
\hline Week 24 & $49(48,51)$ & $49(46,55)$ & $58(51,73)$ & $48(46,53)$ \\
\hline$\Delta$ & $0(-5,1)$ & $0(-5,1)$ & $7(1,13)^{a, b}$ & $0(-3,2)$ \\
\hline$\Delta(\%)$ & $0.0(-8.9,2.1)$ & $0.0(-7.6,1.9)$ & $14.7(2.6,22.1)^{a, b}$ & $0.0(-6.2,3.9)$ \\
\hline \multicolumn{5}{|c|}{ Fasting blood glucose $(\mathrm{mmol} / \mathrm{l})$} \\
\hline Baseline & $7.31(6.40,8.27)$ & $7.48(7.15,8.47)$ & $7.67(6.53,8.87)$ & $7.10(6.51,8.18)$ \\
\hline Week 24 & $7.26(5.99,8.17)$ & $7.39(6.79,8.00)$ & $9.10(7.34,10.50)$ & $7.22(6.59,8.44)$ \\
\hline$\Delta$ & $-0.10(-1.32,0.80)$ & $-0.25(-0.78,0.01)$ & $1.07(0.18,2.02)^{b}$ & $0.05(-0.51,0.47)$ \\
\hline$\Delta(\%)$ & $-1.2(-18.1,12.4)$ & $-3.7(-8.4,0.1)$ & $17.0(1.6,25.2)^{b}$ & $0.8(-5.6,7.4)$ \\
\hline \multicolumn{5}{|c|}{$M\left(\mathrm{mg} / \mathrm{kg} \cdot \mathrm{min}^{-1}\right)$} \\
\hline Baseline & $3.42(2.38,3.97)$ & $2.51(2.12,4.10)$ & $2.66(2.15,3.34)$ & $3.21(2.52,3.68)$ \\
\hline Week 24 & $2.79(1.89,3.09)$ & $2.93(2.69,4.47)$ & $2.33(1.18,3.41)$ & $3.55(3.08,4.34)^{c}$ \\
\hline$\Delta$ & $-0.60(-1.24,0.60)$ & $0.29(-0.34,1.30)$ & $-0.58(-1.25,-0.13)$ & $0.53(-0.20,0.92)$ \\
\hline$\Delta(\%)$ & $-17.1(-38.1,17.2)$ & $12.0(-13.0,52.7)$ & $-21.2(-41.0,1.9)$ & $14.6(-6.2,40.3)$ \\
\hline
\end{tabular}

Data represent a median and interquartile range $(\mathrm{Q} 1, \mathrm{Q} 3)$. Glycemia and plasma $\mathrm{HbA}_{1 \mathrm{c}}$ levels were measured in overnight (8-10-h) fasting patients, while glucose disposal rate (M) was evaluated using hyperinsulinemic-euglycemic clamp at baseline and at week 24 . $\Delta$, a difference between week 24 and baseline values, $\Delta$ (\%), a difference between week 24 and baseline values in \% of the baseline value. ${ }^{a}$, b, cSignificant differences compared with Placebo, Pio, and Omega-3, respectively

carbohydrates, which is usually impaired in insulinresistant individuals [27]. No significant differences in the increase in median RQ between the subgroups were observed (Table 3). Therefore, a robust approach based on the evaluation of percent relative cumulative frequency
(PRCF) curves of RQ values was used while pooling all RQ values from all the patients within subgroups. This was done for both fasting and clamp periods (Fig. 3). Provided that the PRCF curve represents normally distributed data, the value of $\mathrm{EC}_{50}$ of PRCF $\left(50^{\text {th }}\right.$ percentile)

Table 3 Energy metabolism and substrate utilization after the intervention. The significance threshold for adjusted $p$-values using the Holm-Bonferroni correction is 0.05

\begin{tabular}{|c|c|c|c|c|}
\hline & Placebo & Pio & Omega-3 & Pio\& Omega-3 \\
\hline REE (kcal/day) & $1585(1410,2157)$ & $1611(1534,1816)$ & $1780(1653,2000)$ & $1566(1503,1733)$ \\
\hline \multicolumn{5}{|l|}{$\mathrm{RQ}$} \\
\hline Fasting state & $0.80(0.77,0.83)$ & $0.79(0.77,0.85)$ & $0.77(0.74,0.83)$ & $0.80(0.76,0.83)$ \\
\hline During clamp & $0.85(0.81,0.91)$ & $0.87(0.83,0.91)$ & $0.86(0.83,0.87)$ & $0.89(0.84,0.91)$ \\
\hline Diff C-F & $0.07(0.02,0.08)$ & $0.06(0.03,0.09)$ & $0.08(0.02,0.11)$ & $0.07(0.06,0.10)$ \\
\hline \multicolumn{5}{|c|}{ Carbohydrate oxidation (mg/kg.min $\left.{ }^{-1}\right)$} \\
\hline Fasting state & $0.77(0.42,1.18)$ & $0.78(0.47,1.39)$ & $0.44(0.17,0.92)$ & $0.66(0.43,1.27)$ \\
\hline During clamp & $1.09(0.65,1.99)$ & $1.15(0.88,1.51)$ & $1.17(1.02,1.32)$ & $1.50(1.17,1.73)$ \\
\hline Diff C-F & $0.59(0.28,0.79)$ & $0.51(0.00,0.71)$ & $0.90(0.29,1.13)$ & $0.77(0.41,0.82)$ \\
\hline \multicolumn{5}{|c|}{ Fat oxidation (mg/kg.min ${ }^{-1}$ ) } \\
\hline Fasting state & $0.80(0.52,0.88)$ & $0.70(0.31,0.80)$ & $0.91(0.50,1.01)$ & $0.61(0.48,0.89)$ \\
\hline During clamp & $0.43(0.29,0.73)$ & $0.34(0.24,0.52)$ & $0.46(0.34,0.61)$ & $0.32(0.13,0.47)$ \\
\hline Diff C-F & $-0.38(-0.43,-0.1)$ & $-0.20(-0.39,-0.08)$ & $-0.31(-0.54,-0.10)$ & $-0.35(-0.40,-0.24)$ \\
\hline \multicolumn{5}{|c|}{ Non-oxidative GDR (mg/kg.min-1) } \\
\hline & $1.47(0.87,2.00)$ & $1.80(1.28,2.51)$ & $0.60(0.05,1.74)$ & $2.32(1.46,2.81)$ \\
\hline
\end{tabular}

Data represent a median and interquartile range $(\mathrm{Q} 1, \mathrm{Q} 3)$. Data were obtained using indirect calorimetry performed in conjunction with hyperinsulinemiceuglycemic clamp at week 24 (see Table 2). Differences between parameters measured during the clamp and fasting state are also shown (Diff C-F). REE, resting energy expenditure; RQ, respiratory quotient; GDR, glucose disposal rate (indirect calorimetry). For evaluation of the effect of respective interventions on RQ (metabolic flexibility), see also Fig. 3. Non-oxidative glucose GDR was calculated as described in Methods. No significant differences between subgroups were found 


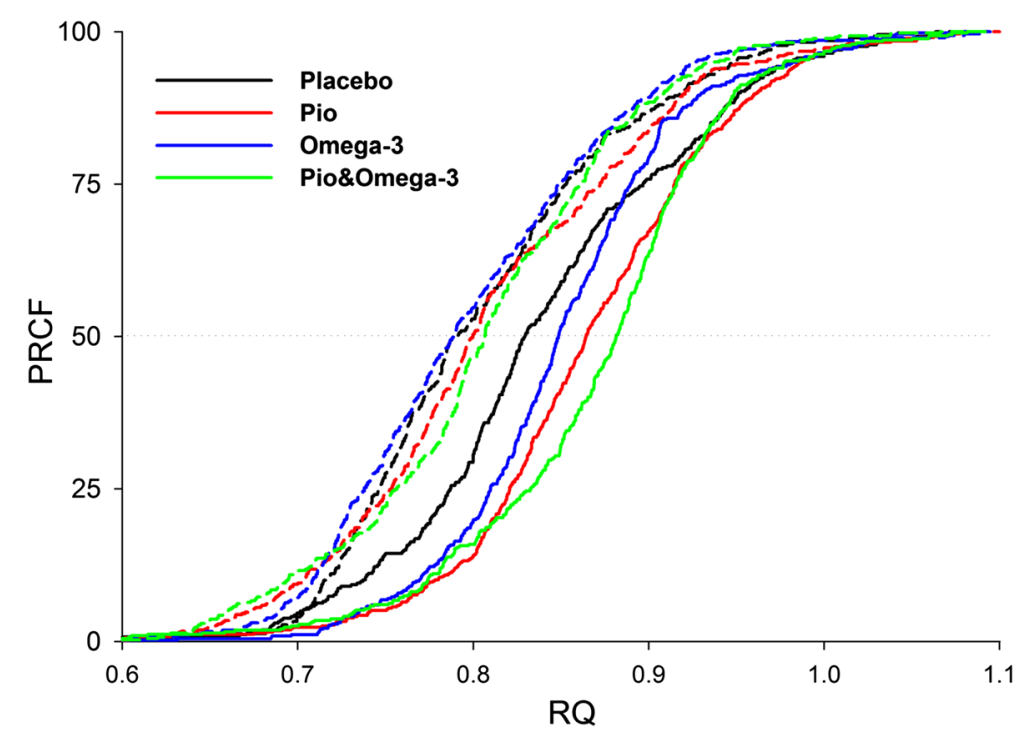

Fig. 3 Metabolic flexibility after the interventions. RQ data from indirect calorimetry at week 24 (see Table 3) were used to construct PRCF curves, each of which represents data ( 400) pooled from all patients in the given subgroup either in fasting state (dashed lines) or during clamp (solid lines). $\mathrm{RQ}$ values corresponding to $\mathrm{EC}_{50}\left(50^{\text {th }}\right.$ percentile value) on each of the curves were obtained and the difference between this $\mathrm{RQ}$ value assessed during the clamp and fasting, respectively, was used as a marker of metabolic flexibility to glucose (PRCF curve shift; Placebo, 0.04; Pio, 0.06; Omega-3, 0.06; Pio\& Omega-3, 0.07)

corresponds to a mean $\mathrm{RQ}$ value, while these curves also allow to identify differences that may exist at either lower or upper levels of RQ range [28]. During the clamp, PRCF curves within each subgroup shifted to the right (i.e., towards glucose oxidation), documenting various degrees of metabolic flexibility to glucose. Compared to Placebo, metabolic flexibility was improved by all interventions in the following order of effect: Pio $<$ Omega-3 $<$ Pio\& Omega-3 (see the PRCF curve shifts in the legend to Fig. 3).

\section{Postprandial metabolism}

A meal test was performed at baseline and at week 24, allowing for assessment of the effects on postprandial metabolism of both glucose and lipids, and of the insulin response to a carbohydrate load. Transient increases in serum glucose, C-peptide, NEFA and triacylglycerol (and insulin; not shown) levels triggered by a standard breakfast were followed during $120 \mathrm{~min}$. Data were expressed as AUC (Fig. 4). At baseline, no significant differences between the subgroups were observed; at week 24, Pio\& Omega-3 subgroup showed faster metabolism of triacylglycerol (lower AUC) compared with both Placebo and Omega-3 ( $p=0.04$; not shown). In the subgroup analysis by the effect of the intervention, several significant results were obtained. Thus, the difference between AUC at week 24 and baseline ( $\triangle \mathrm{AUC}$ ) for glucose was higher in Omega-3 compared with the other subgroups (Fig. 4a; except for Omega-3 vs. Pio\& Omega-3, $p=0.06$ ), suggesting a deterioration of glucose metabolism. In the case of C-peptide (and insulin; not shown), no significant differences between the interventions were found (Fig. 4b). Postprandial metabolism of NEFA was accelerated in response to Pio\& Omega-3, as documented by the lower $\triangle \mathrm{AUC}$ in this subgroup compared with both Placebo and Omega-3 (Fig. 4c). Regarding the metabolism of triacylglycerols (Fig. 4d), $\triangle$ AUC was similar in the Placebo, Pio and Omega-3 subgroup, while it was decreased in response to Pio\& Omega-3.

Previous studies showed an anti-inflammatory effect of EPA + DHA at the level of some plasma cytokines in the postprandial state [29]. Therefore, various antiinflammatory (IL-1RA and IL-10) and pro-inflammatory cytokines (MCP-1, CRP, TNF- $\alpha$ and IL-6), as well as proteins involved in cell adhesion (sVCAM-1, sICAM-1, sE-selectin, sP-selectin, sPECAM-1) and neovascularization (sCD105) were evaluated in serum samples collected at $120 \mathrm{~min}$ of the test, both at baseline and week 24 . Except for sP-selectin, no significant effects of the interventions were observed (see Additional file 3).

\section{Discussion}

We show here that a 6-month-combined intervention using a relatively low dose of pioglitazone and a dose of $\mathrm{EPA}+\mathrm{DHA}$, which is within the range that is approved for treatment of hypertriacylglycerolemia [13], exerts additive beneficial effects on metabolism of both NEFA and triacylglycerols in T2D patients (Fig. 4). All the patients were already receiving metformin therapy and well compensated regarding glycemic control at the baseline, 


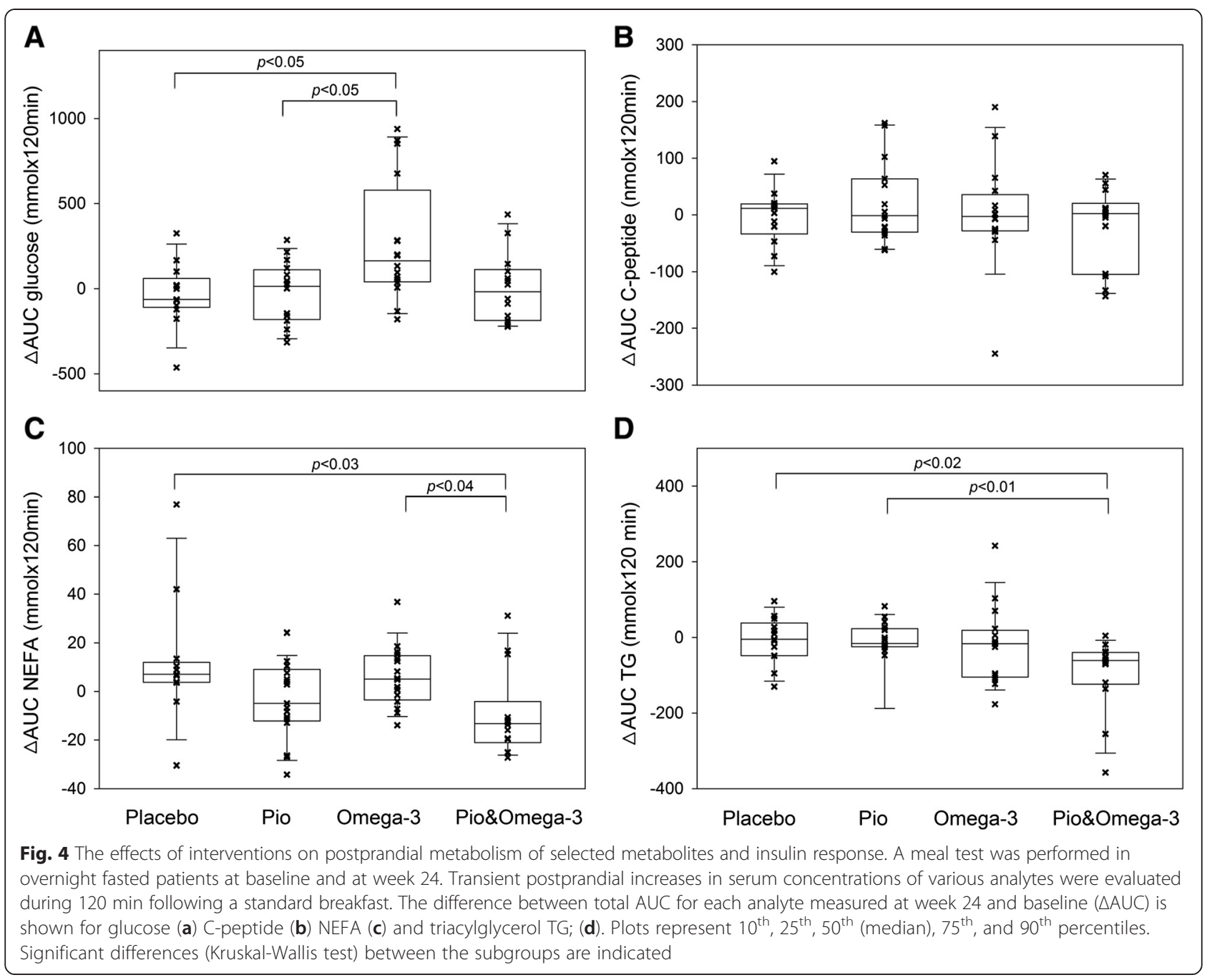

and most of them were obese, thus representing a typical population of patients treated for T2D.

In spite of the triacylglycerol-lowering effect of EPA + DHA, shown in many studies, including those in patients with T2D, as well as the hypolipidemic effects of thiazolidinediones (see Background), fasting serum triacylglycerol levels were not significantly affected by any of the studied interventions. It is likely that the background metformin therapy, which was shown to improve dyslipidemia in patients with T2D [30], could mask the effect of the tested interventions. This is also consistent with the notion that most of the studies demonstrating the triacylglycerol-lowering effect of EPA + DHA in the patients with T2D were performed before the beginning of the metformin era as well as the use of thiazolidinedione therapy of these patients. Moreover, metformin could also mask the additive triacylglycerol-lowering effects of $\mathrm{EPA}+\mathrm{DHA}$ in T2D dyslipidemic patients under statin therapy, which was observed before [31] but not in our study (not shown). Nevertheless, the additive improvement in metabolism of both NEFA and triacylglycerols by the combined intervention found using the meal test in this study (Fig. 4) suggests that increased intake of EPA + DHA could reduce the cardiovascular risk even in T2D patients treated with metformin. This complex effect of the combined intervention is of clinical relevance because increased postprandial triacylglycerolemia represents an independent risk factor of cardiovascular disease in T2D patients $[10,17]$. The mechanisms behind the effect of the combined intervention on metabolism of NEFA and triacylglycerols require clarification. It is likely that PPAR $\alpha$-mediated catabolism of fatty acids [12] and/or their trapping in adipose tissue, i.e., the biochemical activities that are possibly altered in the patients (reviewed in [32]), could contribute to the NEFA-lowering effect. Regarding the beneficial effect on metabolism of triacyglycerols, depression of the rate of VLDL-triacyglycerol secretion from the liver should be considered [7, 33]. 
That all the patients were well-controlled under metformin therapy could also affect other results of the study. First, it could explain why no effect on proteins linked to inflammation (anti - inflammatory cytokines, namely IL-1RA, IL-10, and pro-inflammatory cytokines, namely MCP-1, CRP, TNF- $\alpha$, IL-6) was observed (Additional file 3), since metformin is known to exert anti-inflammatory action [3]. Indeed cytokine and adhesion molecule concentrations (cVCAM-1, sICAM-1, sE-selectin, sPECAM-1) were similar to those reported for healthy male subjects of different ages [34]. Importantly, no deleterious effects of any of the interventions on the markers of oxidative stress (SOD, TBARS, ratio GSSG/ GSH) were observed (Table 1), possibly, due to the antioxidant effect of metformin [4]. Similarly, also insulin secretion remained unaffected by the interventions (see Results, Postprandial metabolism).

Second, only limited additional benefits of pioglitazone and/or Omega-3 may be expected in metformin treated
T2D patients. In fact, Omega-3 alone marginally impaired markers of glycemic control $\left(\mathrm{HbA}_{1 \mathrm{c}}\right.$ levels and fasting glycemia; Table 2) and glucose metabolism in the meal test (Fig. 4), but did not diminish insulin sensitivity (M) evaluated using a hyperinsulinemiceuglycemic clamp (Table 2). These results are compatible with a model in which EPA + DHA per se do not deteriorate glucose utilization when glucose represents the main energy fuel (Fig. 5, Clamp). However, when the supply of both carbohydrates and lipids is increased (e.g., during the meal test; Fig. 5, Fed), or when fatty acids liberated from adipose tissue represent the main energy fuel (Fig. 5, Fasting), glucose utilization is inhibited by multiple mechanisms involved in the Randle cycle (reviewed in [35]) reflecting the PPAR $\alpha$-mediated stimulation of fatty acid oxidation by EPA + DHA [12] This would lead to the observed subtle deterioration of glucose metabolism by Omega-3. Metabolic changes in skeletal muscle, the main site of glucose utilization,

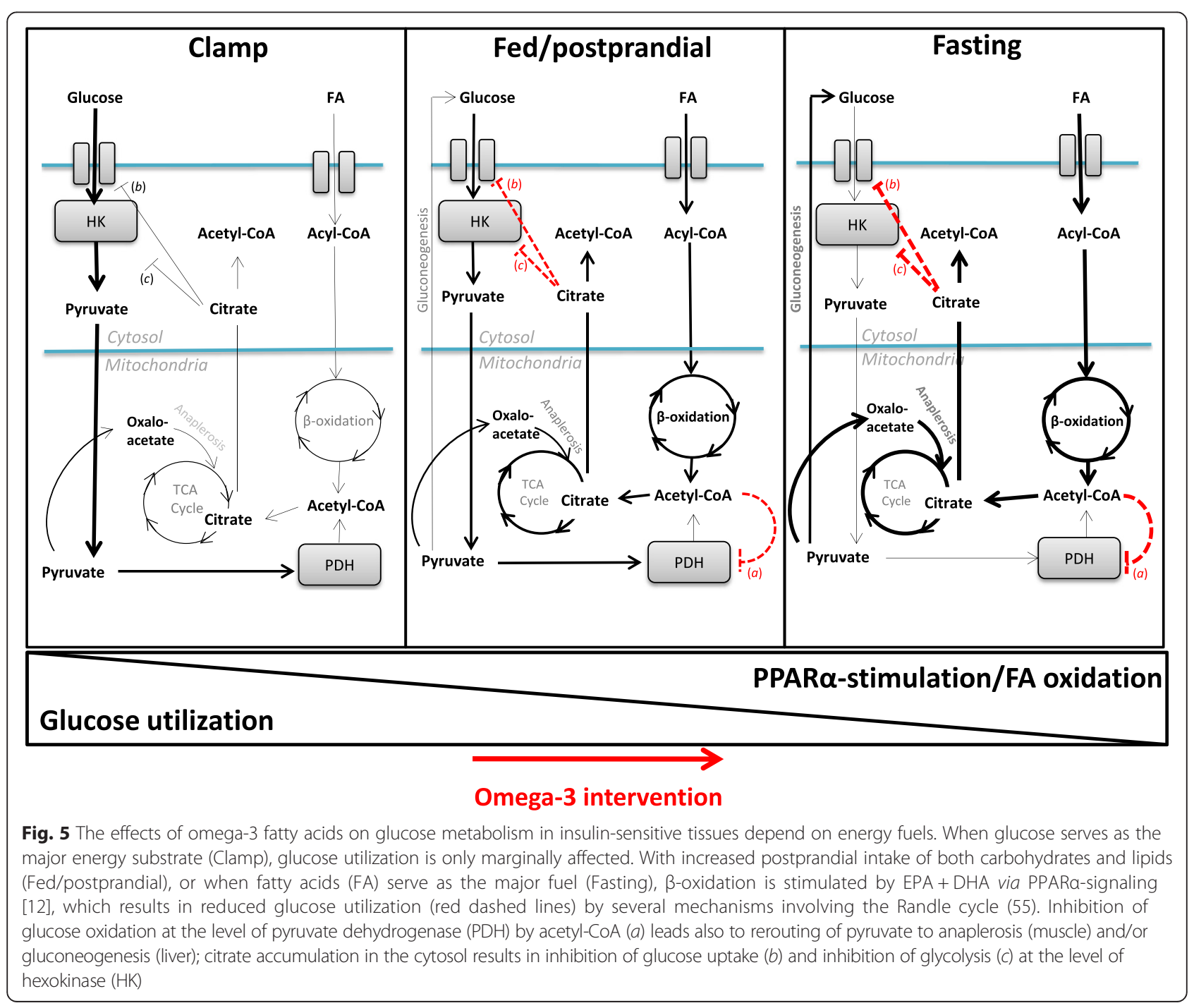


probably play a major role. That fasted glycemia is selectively increased by Omega-3 (Table 2) could also reflect increased hepatic gluconeogenesis stimulated in face of enhanced fatty acid oxidation [36]. Both decreased postprandial metabolism of glucose (Fig. 4) and elevated glycemia in fasted state could contribute to raised $\mathrm{HbA}_{1 \mathrm{c}}$ levels in the Omega-3 subgroup (Table 2). Thus, our results also help to clarify some controversies regarding the effects of EPA + DHA on glucose homeostasis in T2D patients (see Background).

The negative effects of Omega-3 on glycemic control and glucose metabolism were prevented by Pio. Insulin sensitivity was increased by Pio\&Omega-3, and tended to be improved by Pio compared with Omega-3 (Table 2), which is also in agreement with the induction of adiponectin by both Pio\& Omega- 3 and Pio (Table 1). These results were consistent with changes in metabolic flexibility to glucose evaluated using indirect calorimetry during the hyperinsulinemiceuglycemic clamp at week 24 (Fig. 3), since this parameter closely reflects whole-body glucose uptake [27]. Thus, all the interventions, and especially Pio\& Omega-3, increased metabolic flexibility. These results are consistent with the above model and also with our previous study showing additive improvement in metabolic flexibility [20] and insulin sensitivity [7] by combined interventions using EPA + DHA and thiazolidinediones in dietary obese mice. In both the animal experiments and the present clinical trial, the robust PRCF analysis of RQ was used. This approach, which revealed here subtle differences in metabolic flexibility, has not been applied in humans before.

Few studies were conducted to characterize possible modulation of metabolic flexibility by EPA + DHA in T2D patients, and very little is known about the effects of combined interventions using EPA + DHA and pharmaceuticals. It has been shown that EPA + DHA administered as a 4-h lipid infusion resulted in a marginal improvement of metabolic flexibility without affecting insulin sensitivity [37]. Over a 9-weekperiod, dietary EPA + DHA exerted a transient improvement of glucose utilization followed by a shift from glucose to lipid catabolism, but the effect on metabolic flexibility is difficult to assess from these data since a relatively large volume $(20 \mathrm{ml})$ of crude fish oil containing different lipid fractions besides EPA + DHA was used [38]. Thus, our study is unique regarding the use of a complex methodological approach including the indirect calorimetry, clamp as well as a meal test, which allowed us to demonstrate the additive improvements in metabolic flexibility to glucose, and namely in the postprandial lipid metabolism, by pioglitazone in combination with highly purified EPA + DHA.
Evaluation of serum levels of both pioglitazone and EPA + DHA increased the power of the study by controlling the adherence to the therapy, and enabled a more detailed analysis of the measured parameters relative to the changes in Omega-3 PhL Index. However, only weak correlations $(p<0.1)$ were detected when an increase in $\mathrm{HbA}_{1 \mathrm{c}}$ levels or a decrease in NEFA levels was examined (see Additional file 4). Further studies are needed to analyze the mechanisms behind the metabolic effects of interventions observed in our study, including the evaluation of changes in muscle glycogen content during the clamp. In this context, muscle glycogen was measured only in the fasting state and no differences between the subgroups were found (Additional file 1).

In spite of the fact that pioglitazone is a well-established pharmaceutical with lasting insulin-sensitizing effects, and despite its other beneficial effects in patients with T2D, its clinical use has declined recently due to the risk of the side-effects (see Background). Importantly, at least some of these concerns have been disproved recently. Namely, it has been demonstrated that the cumulative use of pioglitazone or rosiglitazone was not associated with the incidence of bladder cancer [39]. The results of our study document beneficial effects of a relatively low dose of pioglitazone on lipid metabolism when pioglitazone was used as part of the combined intervention with $n-3$ fatty acids. This observation is relevant for reducing the risk of the side-effects of pioglitazone under clinical settings.

\section{Conclusions}

In overweight/obese T2D patients on stable metformin therapy, and in spite of the modest negative effect of Omega-3 on glycemic control and postprandial glucose metabolism, no adverse effect on insulin sensitivity was observed. In response to the combined intervention using Pio\& Omega-3, the negative effect of Omega-3 on glucose metabolism was avoided, insulin sensitivity increased, and lipid metabolism was additively improved. Thus, typical T2D patients may be advised to increase their EPA + DHA intake, either in the form of dietary supplements or sea food and fish, in order to increase the efficacy of pharmacotherapies and to prevent diseases linked to inflammation as well as cardiovascular disease, providing that glycemic control is closely monitored. EPA + DHA are likely to bring benefits on cardiovascular health of diabetic patients.

\section{Additional files}

Additional file 1: Liver and muscle lipid content before and after the intervention and muscle glycogen content after the intervention. (DOCX $44 \mathrm{~kb}$ )

Additional file 2: The content of linoleic acid (LA) in serum phospholipids. (DOCX $94 \mathrm{~kb}$ ) 


\section{Additional file 3: Serum levels of inflammatory markers in the postprandial state. (DOCX $39 \mathrm{~kb})$}

Additional file 4: Correlations between changes in Omega-3 PhL Index and changes in selected variables in response to interventions. (DOCX $22909 \mathrm{~kb}$ )

\section{Abbreviations}

$\triangle$ : difference between week 24 and baseline values; AUC: area under the curve; BMl: body mass index; DHA: docosahexaenoic acid; EPA: eicosapentaenoic acid; GDR: glucose disposal rate (indirect calorimetry); GSH: reduced glutathione; GSSG: oxidized glutathione; $\mathrm{HbA}_{1 c}$ : hemoglobin $\mathrm{A}_{1 \mathrm{c}}$ IL: interleukin; IQR: interquartile range; LA: linoleic acid (18:2 n-6); M: glucose disposal rate (hyperinsulinemic-euglycemic clamp); NEFA: non-esterified fatty acids; Omega-3: EPA + DHA concentrate (5 g/day) supplementation; Omega-3 PhL Index: relative concentration of EPA + DHA in plasma phospholipids; Pio: pioglitazone (15 mg/day) intervention; Pio\& Omega3: pioglitazone (15 mg/day) intervention combined with EPA + DHA concentrate ( $5 \mathrm{~g} /$ day) supplementation; PPAR: peroxisome proliferatoractivated receptor; PRCF: percent relative cumulative frequency; REE: resting energy expenditure; RQ: respiratory quotient; SOD: superoxide dismutase; T2D: type 2 diabetes; TBARS: thiobarbituric acid reactive substances; $\mathrm{VCO}_{2}$ : carbon dioxide production; $\mathrm{VO}_{2}$ : oxygen consumption.

\section{Competing interests}

The authors declare that they have no competing interests associated with this study.

\section{Authors' contributions}

MB, PCC, JK Sr., TP designed the study and wrote the manuscript. JV, JK Jr. and TP recruited the patients and acquired the clinical data. PJ, OK, OH, HM, LK, OO, VS, JT, MH, AS, PF, KB, MR, JO, GSC, and JJ were involved in the acquisition and analysis of the laboratory data. AG and EF carried out the statistical analyses and interpretation of the data. All authors had full access to data and approved the manuscript for publication. JK Sr. and TP wrote the grant application and are the guarantors.

\section{Acknowledgements}

This work was supported by the grant from the Ministry of Health of the Czech Republic (NT13763-4). The gift of Placebo and Omega-3 capsules by EPAX AS, Aalesund, Norway is acknowledged. We thank Prof. Erik Richter for the help regarding muscle biopsies and Dr. Vera Lanska for the help with statistical analysis.

\section{Author details}

${ }^{1}$ Institute for Clinical and Experimental Medicine, Prague, Czech Republic. ${ }^{2}$ Department of Adipose Tissue Biology, Institute of Physiology of the Czech Academy of Sciences, Prague, Czech Republic. ${ }^{3}$ Human Development \& Health Academic Unit, Faculty of Medicine, University of Southampton, Southampton, UK. ${ }^{4}$ Department of Mathematical Analysis and Applications of Mathematics, Faculty of Science, Palacky University, Olomouc, Czech Republic. ${ }^{5}$ Department of Physical Performance, Norwegian School of Sport Sciences, Oslo, Norway. 'Silentia AS, Svelvik, Norway.

Received: 28 July 2015 Accepted: 25 November 2015 Published online: 02 December 2015

\section{References}

1. Inzucchi SE, Bergenstal RM, Buse JB, Diamant M, Ferrannini E, Nauck M, et al. Management of hyperglycaemia in type 2 diabetes, 2015: a patient-centred approach. Update to a Position Statement of the American Diabetes Association and the European Association for the Study of Diabetes. Diabetologia. 2015;58:429-42.

2. American DA. Standards of medical care in diabetes-2014. Diabetes Care. 2014;37 Suppl 1:S14-80.

3. Kim J, Kwak HJ, Cha JY, Jeong YS, Rhee SD, Kim KR, et al. Metformin suppresses lipopolysaccharide (LPS)-induced inflammatory response in murine macrophages via activating transcription factor-3 (ATF-3) induction. J Biol Chem. 2014;289:23246-55.

4. Mirmiranpour H, Mousavizadeh M, Noshad S, Ghavami M, Ebadi M, Ghasemiesfe $M$, et al. Comparative effects of pioglitazone and metformin on oxidative stress markers in newly diagnosed type 2 diabetes patients: a randomized clinical trial. J Diabetes Complications. 2013;27:501-7.

5. Rodriguez A, Reviriego J, Karamanos V, del Canizo FJ, Vlachogiannis N, Drossinos $V$, et al. Management of cardiovascular risk factors with pioglitazone combination therapies in type 2 diabetes: an observational cohort study. Cardiovasc Diabetol. 2011;10:18.

6. Kus V, Flachs P, Kuda O, Bardova K, Janovska P, Svobodova M, et al. Unmasking Differential Effects of Rosiglitazone and Pioglitazone in the Combination Treatment with n-3 Fatty Acids in Mice Fed a High-Fat Diet. Plos One. 2011;6:e27126-7.

7. Kuda O, Jelenik T, Jilkova Z, Flachs P, Rossmeisl M, Hensler M, et al. n-3 Fatty acids and rosiglitazone improve insulin sensitivity through additive stimulatory effects on muscle glycogen synthesis in mice fed a high-fat diet. Diabetologia. 2009;52:941-51.

8. Yang J, Vallarino C, Bron M, Perez A, Liang H, Joseph G, et al. A comparison of all-cause mortality with pioglitazone and insulin in type 2 diabetes: an expanded analysis from a retrospective cohort study. Curr Med Res Opin. 2014;30:2223-31

9. Anagnostis P, Karras SN. Should we stop prescribing pioglitazone? Br J Clin Pharmacol. 2014;78:438-9.

10. Huxley R, Barzi F, Woodward M. Excess risk of fatal coronary heart disease associated with diabetes in men and women: meta-analysis of 37 prospective cohort studies. BMJ. 2006;332:73-8.

11. Flachs $P$, Rossmeisl M, Kopecky J. The Effect of $n-3$ Fatty Acids on Glucose Homeostasis and Insulin Sensivity. Physiol Res. 2014;63:93-118.

12. Rakhshandehroo M, Hooiveld G, Muller M, Kersten S. Comparative analysis of gene regulation by the transcription factor PPARalpha between mouse and human. Plos One. 2009;4:e6796.

13. Kris-Etherton PM, Harris WS, Appel $\amalg$, Association AHANCAH. Omega-3 fatty acids and cardiovascular disease: new recommendations from the American Heart Association. Arterioscler Thromb Vasc Biol. 2003;23:151-2.

14. Scorletti E, Bhatia L, McCormick KG, Clough GF, Nash K, Hodson L, et al. Effects of purified eicosapentaenoic and docosahexaenoic acids in nonalcoholic fatty liver disease: results from the Welcome* study. Hepatology. 2014;60:1211-21.

15. Mozaffarian D, Lemaitre RN, King IB, Song $X$, Huang $H$, Sacks FM, et al Plasma phospholipid long-chain omega-3 fatty acids and total and cause-specific mortality in older adults: a cohort study. Ann Intern Med. 2013;158:515-25.

16. Calder PC, Yagoob P. Omega-3 (n-3) fatty acids, cardiovascular disease and stability of atherosclerotic plaques. Cell Mol Biol (Noisy-le-Grand). 2010;56:28-37.

17. Authors/Task Force M, Ryden L, Grant PJ, Anker SD, Berne C, Cosentino F, et al. ESC Guidelines on diabetes, pre-diabetes, and cardiovascular diseases developed in collaboration with the EASD: the Task Force on diabetes, pre-diabetes, and cardiovascular diseases of the European Society of Cardiology (ESC) and developed in collaboration with the European Association for the Study of Diabetes (EASD). Eur Heart J. 2013;34:3035-87.

18. Kromhout D, Geleijnse JM, de Goede J, Oude Griep LM, Mulder BJ, de Boer MJ, et al. n-3 fatty acids, ventricular arrhythmia-related events, and fatal myocardial infarction in postmyocardial infarction patients with diabetes. Diabetes Care. 2011;34:2515-20.

19. Dangardt F, Chen Y, Gronowitz E, Dahlgren J, Friberg P, Strandvik B. High physiological omega-3 Fatty Acid supplementation affects muscle Fatty Acid composition and glucose and insulin homeostasis in obese adolescents. J Nutr Metab. 2012;2012:395757.

20. Horakova O, Medrikova D, van Schothorst EM, Bunschoten A, Flachs P, Kus $V$, et al. Preservation of Metabolic Flexibility in Skeletal Muscle by a Combined Use of n-3 PUFA and Rosiglitazone in Dietary Obese Mice. Plos One. 2012;7:e43764.

21. Pelikanova T, Smrckova I, Krizova J, Stribrna J, Lanska V. Effects of insulin and lipid emulsion on renal haemodynamics and renal sodium handling in IDDM patients. Diabetologia. 1996;39:1074-82.

22. Golay A, DeFronzo RA, Ferrannini E, Simonson DC, Thorin D, Acheson K, et al, Oxidative and non-oxidative glucose metabolism in non-obese type 2 (noninsulin-dependent) diabetic patients. Diabetologia. 1988;31:585-91.

23. Ejsing CS, Duchoslav E, Sampaio J, Simons K, Bonner R, Thiele C, et al. Automated identification and quantification of glycerophospholipid molecular species by multiple precursor ion scanning. Anal Chem. 2006;78: 6202-14.

24. Kahleova H, Matoulek M, Malinska H, Oliyarnyk O, Kazdova L, Neskudla T, et al. Vegetarian diet improves insulin resistance and oxidative stress markers more 
than conventional diet in subjects with Type 2 diabetes. Diabet Med. 2011;28: 549-59.

25. Jagadeesh B, Bharathi DV, Pankaj C, Narayana VS, Venkateswarulu V. Development and validation of highly selective and robust method for simultaneous estimation of pioglitazone, hydroxypioglitazone and metformin in human plasma by LC-MS/MS: application to a pharmacokinetic study. J Chromatogr B Analyt Technol Biomed Life Sci. 2013;930:136-45.

26. Harris WS, Von Schacky C. The Omega-3 Index: a new risk factor for death from coronary heart disease? Prev Med. 2004;39:212-20.

27. Galgani JE, Moro C, Ravussin E. Metabolic flexibility and insulin resistance. Am J Physiol Endocrinol Metab. 2008;295:E1009-1017.

28. Riachi M, Himms-Hagen J, Harper ME. Percent relative cumulative frequency analysis in indirect calorimetry: application to studies of transgenic mice. Can J Physiol Pharmacol. 2004;82:1075-83.

29. Burdge GC, Calder PC. Plasma cytokine response during the postprandial period: a potential causal process in vascular disease? Br J Nutr. 2005;93:3-9.

30. Wulffele MG, Kooy A, de Zeeuw D, Stehouwer CD, Gansevoort RT. The effect of metformin on blood pressure, plasma cholesterol and triglycerides in type 2 diabetes mellitus: a systematic review. J Intern Med. 2004;256:1-14.

31. Chan DC, Watts GF, Mori TA, Barrett PH, Beilin LJ, Redgrave TG. Factorial study of the effects of atorvastatin and fish oil on dyslipidaemia in visceral obesity. Eur J Clin Invest. 2002;32:429-36.

32. Flachs P, Rossmeisl M, Kuda O, Kopecky J. Stimulation of mitochondrial oxidative capacity in white fat independent of UCP1: A key to lean phenotype. Biochim Biophys Acta. 1831;2013:986-1003.

33. Wong AT, Chan DC, Ooi EM, Ng TW, Watts GF, Barrett PH. Omega-3 fatty acid ethyl ester supplementation decreases very-low-density lipoprotein triacylglycerol secretion in obese men. Clin Sci (Lond). 2013;125:45-51.

34. Miles EA, Rees D, Banerjee T, Cazzola R, Lewis S, Wood R, et al. Age-related increases in circulating inflammatory markers in men are independent of BMI, blood pressure and blood lipid concentrations. Atherosclerosis. 2008; 196:298-305.

35. Hue L, Taegtmeyer $H$. The Randle cycle revisited: a new head for an old hat. Am J Physiol Endocrinol Metab. 2009;297:E578-91.

36. Williamson JR, Kreisberg RA, Felts PW. Mechanism for the stimulation of gluconeogenesis by fatty acids in perfused rat liver. Proc Natl Acad Sci U S A. $1966 ; 56: 247-54$

37. Mostad IL, Bjerve KS, Basu S, Sutton P, Frayn KN, Grill V. Addition of n-3 fatty acids to a 4-hour lipid infusion does not affect insulin sensitivity, insulin secretion, or markers of oxidative stress in subjects with type 2 diabetes mellitus. Metabolism. 2009;58:1753-61.

38. Mostad IL, Bjerve KS, Bjorgaas MR, Lydersen S, Grill V. Effects of n-3 fatty acids in subjects with type 2 diabetes: reduction of insulin sensitivity and time-dependent alteration from carbohydrate to fat oxidation. Am J Clin Nutr. 2006:84:540-50

39. Levin D, Bell S, Sund R, Hartikainen SA, Tuomilehto J, Pukkala E, et al. Pioglitazone and bladder cancer risk: a multipopulation pooled, cumulative exposure analysis. Diabetologia. 2015;58:493-504.

\section{Submit your next manuscript to BioMed Central and we will help you at every step:}

- We accept pre-submission inquiries

- Our selector tool helps you to find the most relevant journal

- We provide round the clock customer support

- Convenient online submission

- Thorough peer review

- Inclusion in PubMed and all major indexing services

- Maximum visibility for your research

Submit your manuscript at www biomedcentral com/submit 